\title{
Home Birth in the Era of COVID-19: Counseling and Preparation for Pregnant Persons Living with HIV
}

\author{
Ashish Premkumar, MD ${ }^{1}$ Irina Cassimatis, $\mathrm{MD}^{1}$ Saba H. Berhie, $\mathrm{MD}^{1}$ Jennifer Jao, MD, $\mathrm{MPH}^{2,3}$ \\ Susan E. Cohn, MD, MPH ${ }^{3}$ Sarah H. Sutton, MD ${ }^{3}$ Brianne Condron, RN ${ }^{1}$ \\ Jordan Levesque, MSW, LCSW ${ }^{1}$ Patricia M. Garcia, MD, MPH ${ }^{1}$ Emily S. Miller, MD, MPH ${ }^{1}$ \\ Lynn M. Yee, MD, MPH ${ }^{1}$
}

1 Department of Obstetrics and Gynecology, Feinberg School of

Medicine, Northwestern University, Chicago, Illinois

2 Department of Pediatrics, Feinberg School of Medicine,

Northwestern University, Chicago, Illinois

${ }^{3}$ Department of Internal Medicine, Feinberg School of Medicine,

Northwestern University, Chicago, Illinois

Am J Perinatol 2020;37:1038-1043.

\begin{abstract}
Keywords

- out-of-hospital birth

- HIV

- perinatal transmission

- COVID-19

With the coronavirus disease 2019 (COVID-19) pandemic in the United States, a majority of states have instituted "shelter-in-place" policies effectively quarantining individuals-including pregnant persons-in their homes. Given the concern for COVID19 acquisition in health care settings, pregnant persons with high-risk pregnanciessuch as persons living with HIV (PLHIV)-are increasingly investigating the option of a home birth. Although we strongly recommend hospital birth for PLHIV, we discuss our experience and recommendations for counseling and preparation of pregnant PLHIV who may be considering home birth or at risk for unintentional home birth due to the pandemic. We also discuss issues associated with implementing a risk mitigation strategy involving high-risk births occurring at home during a pandemic.
\end{abstract}

\section{Key Points}

- Coronavirus disease 2019 pandemic has increased interest in home birth.

- Women living with HIV are pursuing home birth.

- Safe planning is paramount for women living with HIV desiring home birth, despite recommending against the practice.

Since the first cases of coronavirus disease 2019 (COVID-19) in December 2019 in Wuhan, China, the spread of the virus has ushered in the largest global health emergency of the $21 \mathrm{st}$ century. ${ }^{1,2}$ Estimates as of May 3, 2020 demonstrate that 3.3 million individuals have been infected, with a case-fatality rate of approximately $7.1 \%$ from serious complications of the infection. ${ }^{3}$ Limited experience with the clinical course among pregnant women with COVID-19, particularly in the mid-to-

received

May 3, 2020

accepted after revision

May 5, 2020

published online

June 4, 2020 late third trimester, have been published. ${ }^{4-17}$ Existing data regarding perinatal COVID-19 infection have reported requirements for mechanical ventilation, with need for extracorporeal membranous oxygenation support, as well as maternal death. ${ }^{6,9,12,18,19}$ Furthermore, although case series postulate the potential for perinatal transmission of COVID-19, ${ }^{10,20-22}$ this has not yet clearly been demonstrated. Nonetheless, maternal COVID-19 acquisition has been associated with a
Copyright $\odot 2020$ by Thieme Medical Publishers, Inc., 333 Seventh Avenue, New York, NY 10001, USA. Tel: $+1(212) 760-0888$.
DOI https://doi.org/ 10.1055/s-0040-1712513. ISSN 0735-1631. 
high incidence of preterm birth, fetal distress, delivery via cesarean, and perinatal loss. ${ }^{23-29}$

Given the spread of COVID-19, many states have enacted "shelter-in-place" policies, effectively quarantining individuals to their homes to reduce the chance of acquiring or spreading the virus in community spaces, which may limit health care access for some individuals. Moreover, given concerns of COVID-19 transmission on labor and delivery floors and hospital policies emphasizing the limitation of visitors, some pregnant persons have explored the option of pursuing a home birth. $^{30-32}$ In our clinical experience as one of the largest centers providing perinatal care for pregnant persons living with HIV (PLHIV) in Illinois, we have noted robust community discussions regarding the option of a home birth to avoid the potential for acquiring COVID-19. Pregnant PLHIV have reported to our medical team, perinatal case managers, and on social media about the fear of hospital birth due to perceived greater risk of COVID-19 acquisition, fear that of reduced hospital access due to shelter-in-place restrictions, concerns over potential visitor limitations, and worry that they may receive poor quality care due to overwhelmed medical systems. Our center has already fielded patient queries about these fears and their consideration of home birth as either an active decision or as an unintended potential outcome of limited mobility under shelter-in-place rules.

In this call to action, we briefly review the literature on home birth in the United States and offer recommendations on counseling and peripartum management of pregnant persons considering home birth, focusing our discussion on pregnant PLHIV and the unique considerations of an ongoing pandemic.

\section{Home Birth in the United States}

The COVID-19 pandemic has focused attention on a potential rise in the number of pregnant people interested in pursuing home birth or who may be at increased risk for an unintentional home birth. Multiple news media outlets have noted these concerns and discussions among doulas, midwives, and researchers in reproductive health have noted a perceived increase in demands for home birth resources. ${ }^{30-32}$

Although planned home birth among a self-selected U.S. population has been associated with a reduction in the performance of operative vaginal delivery, cesarean delivery, and blood transfusion, it has been associated with several adverse maternal outcomes, including protracted labor, need for transfer to a higher level facility for intrapartum management, and higher order perineal lacerations. ${ }^{33-35}$ From a neonatal standpoint, home birth is associated with higher frequencies of low Apgar values, neurological dysfunction, and mortality. ${ }^{34-42}$ Statements from the American College of Obstetricians and Gynecologists (ACOG), American Academy of Pediatrics (AAP), and the National Academy of Medicine noted the limitations in the aforementioned studies. ${ }^{41,43,44}$ ACOG, AAP, the American College of Nurse-Midwives, and the Society for Maternal-Fetal Medicine support a personfirst, shared decision-making approach to counseling, recommending against home birth and discussing the potential risks and benefits. ${ }^{43,45}$
Proponents of home birth in the United States have recommended the implementation of additional measures to improve care provided at the time of a home birth. These include risk stratification for women attempting home birth; systems of consultation between obstetricians and home birth midwives; methods of consolidating transport services to and from hospitals; and most importantly, the acknowledgment of direct-entry midwives and certified professional midwives within the medical licensure system as birth attendants to ensure appropriate evaluation of their skills. ${ }^{46,47}$ In Illinois, current legislation does not regulate birth attendants practicing outside of the hospital setting. The Home Birth Safety Act (HB2449), currently in review, would allow for both licensure and regulation of home birth attendants. ${ }^{48}$

The risks of COVID-19 spread during hospitalization have led to restrictions on visitors during labor and delivery, which can create isolation and potential disempowerment of the pregnant person. Counseling around home birth must be updated to consider the novel stressors that the pandemic is imposing not only on the health care system, but also on the decisions pregnant persons make regarding the location of their birth. Careful consideration must also be given to the unique barriers pregnant persons experience in the context of limited access to health care and limited mobility due to social distancing and shelter-in-place requirements.

\section{Counseling for Pregnant PLHIV Regarding Reducing Exposures in Health Care Settings}

In our practice, pregnant PLHIV have expressed concerns about exposure to COVID-19 during outpatient and inpatient care. Furthermore, in-person obstetric visits for all patients, including for PLHIV, have been reduced to limit patient exposure. ${ }^{49}$ We have yet to understand the clinical or psychosocial implications of this change and what alterations are seen in the patient-provider relationship. Despite these gaps in knowledge, we recommend health care providers counsel and inform their patients of the inpatient and outpatient practices in place to reduce exposures, as well as to emphasize provider availability even if in-person visits are limited. Patients should be aware that guidance on best practices on labor and delivery units during COVID-19 is being implemented widely to reduce COVID-19 exposures (e.g., use of face masks for all patients and clinical staff; limiting patient-facing interactions with a core group of staff members; and universal COVID-19 nasopharyngeal swab testing, depending on hospital policies). ${ }^{49,50}$

\section{Counseling and Preparation for Pregnant PLHIV Desiring a Home Birth}

Pregnant PLHIV face a variety of risks during the intrapartum period, including the risk of perinatal transmission of HIV. In the setting of maternal viremia, the probability of transmission can be as high as $40 \%$ without appropriate intrapartum measures such as prelabor cesarean delivery and intravenous zidovudine. ${ }^{51-55}$ However, in the setting of viral suppression, the risk of transmission is less than $1 \%{ }^{56}$ To further reduce 
this risk, ACOG recommends avoidance of operative vaginal delivery, placement of fetal scalp electrode, and performance of episiotomy outside of routine obstetrical indications. ${ }^{57}$

From a neonatal perspective, the U.S. Department of Health and Human Services (DHHS) recommend administration of oral zidovudine, with the potential for additional antiretroviral medications (ARVs) based on a neonate's a priori chance of acquiring HIV in utero, as well as avoidance of breastfeeding. ${ }^{58}$ Also, our practice is to advocate for early bathing of the neonate to reduce HIV exposure. Coordination with a pediatric infectious disease specialist is also essential to ensure appropriate completion of time-sensitive issues such as HIV postexposure prophylaxis and testing.

Given these unique needs of pregnant PLHIV and newborns exposed to HIV, we have devised a checklist of counseling and planning considerations for individuals considering a home birth ( - Table 1). We stand by ACOG's recommendation against home birth, especially among pregnant PLHIV; however, we recognize our strategy as one of harm reduction. Regarding counseling, we support the person-first, shared decision-making perspective adopted by ACOG, specifically discussing the twofold increase in risk of perinatal death and threefold increase in risk of neonatal seizures or serious neurological dysfunction when compared with a hospital-based birth. ${ }^{43}$ We also agree with the emphasis on selecting an appropriate birth attendant, emphasizing a midwife whose education meets the International Confederation of Midwives' Global Standards for Midwifery Education or a physician with training in obstetrics. We recognize that given the harsh legal atmosphere surrounding home birth, as well as the potential high out-ofpocket cost, access to appropriately trained professionals may be limited. ${ }^{59,60}$

We also recommend discussing the risk of intrapartum transmission, specifically emphasizing the need for adherence to ARVs, in consultation with an adult infectious disease specialist and/or a maternal-fetal medicine subspecialist with expertise in perinatal HIV. Furthermore, counseling may include that prolonged rupture of membranes increases the risk of chorioamnionitis, which has been demonstrated to increase the risk of transmission; such counseling may include recommendations for early hospital transfer. ${ }^{61}$ Furthermore, route of delivery should be dictated not only by routine obstetrical indications, but by presence of maternal viremia; an attempt at a home birth should be made only in the setting of viral suppression, with confirmation of viral suppression made at 35 to 37 weeks of gestation as viral rebound occurs in a minority of pregnant PLHIV at term. ${ }^{62}$

Creating a safety plan in case of clinical questions or need for transfer to a higher level facility is paramount in the case of home birth, particularly for pregnant PLHIV. Key areas of concern for hospital-based care providers who are receiving pregnant persons attempting home birth include the lack of clinical information provided by the home birth attendant. ${ }^{63}$ Therefore, having an adult obstetric care provider identified at the hospital antenatally who would facilitate a transfer is essential for a thorough warm handoff, as is identification of a pediatric infectious disease specialist for appropriate care of the newborn. Moreover, ensuring medical records-
Table 1 Components of counseling for pregnant persons living with HIV who are choosing home birth

Timing of counseling
Antepartum
Utilize telemedicine services
to discuss risks of home
birth and perinatal
transmission

Consider additional elements of antenatal care that may affect counseling regarding home birth (e.g., obtaining an obstetrical ultrasound to estimate fetal weight)

Establish a safe, effective connection between home birth care providers, and a local hospital obstetric unit, as well as a pediatric infectious disease physician

At 32 weeks' gestation or greater, provide patient with a prescription for neonatal antiretroviral medications

Obtain HIV viral load at 35-37 weeks' gestation to ensure that a vaginal trial of labor is medically appropriate

\section{Intrapartum}

Administer intravenous zidovudine after shared decision-making discussion with home birth attendant and obstetrician

Use appropriate personal protective equipment to reduce the risk of exposure to bodily fluids

Avoid episiotomy and operative vaginal delivery

\section{Postpartum}

Early bath for the neonate

Avoid methergine if patient is on a protease inhibitor (e.g., darunavir) due to risk of severe hypertension

Avoid breastfeeding

Coordinate appropriate

laboratory testing with pediatric infectious disease (e.g., HIV DNA PCR)

\section{Citations}

ACOG $2017^{43}$

ACOG $2018^{57}$

Boelig et $\mathrm{al}^{49}$

SMFM $2020^{67}$

ACOG $2018^{57}$

Cheyney and

Caughey ${ }^{68}$

Cheyney and

Caughey ${ }^{68}$

U.S. Department of Health and Human Services $2019^{58}$

ACOG $2018^{57}$

Briand et al $^{56}$

ACOG $2018^{57}$

U.S. Department of Health and Human Services $2019^{65}$

Expert opinion ACOG $2018^{57}$

Expert opinion ACOG $2018^{57}$

Abbreviations: American College of Obstetricians and Gynecologists; $\mathrm{PCR}$, polymerase chain reaction. 
complete with routine prenatal laboratories, HIV viral loads, CD4 counts, current ARV regimen, and ultrasounds-are present at the transfer hospital before labor is also crucial for emergency preparedness. Proactive measures on the part of hospital teams, such as outreach to pregnant PLHIV through regular telephone contact, facilitating appropriate transfer to hospitals, or the creation of a telephone hotline for patient questions can be considered. Finally, local and national resources are available for clinical questions pertaining to management of HIV. ${ }^{22,64}$

Regarding intrapartum management for virally suppressed pregnant PLHIV (e.g., viral load less than 1,000 copies/mL), ACOG and the U.S. DHHS state that intravenous zidovudine does not need to be administered to reduce the risk for vertical transmission. ${ }^{57,65}$ An important consideration is that data informing this practice note no difference in frequency of transmission among pregnant PLHIV with viral loads $<400$ copies/mL who did and did not receive intrapartum zidovudine ( 0 vs. $0.6 \%, p=0.17$ ). ${ }^{56}$ However, in our practice, we continue to recommend administration of intravenous zidovudine regardless of viral load. Therefore, a shared decision-making approach should be taken toward administration of intrapartum zidovudine, taking into consideration access to needed supplies in the out-of-hospital setting as well as provider comfort. Furthermore, the pregnant PLIV should continue their oral ARV regimen throughout labor. We also recommend appropriate personal protective equipment be used by home birth attendants, including face masks, goggles, gown, and sterile gloves to reduce risks of HIV exposure.

For postpartum care, in line with ACOG and the U.S. DHHS, we also recommend against breastfeeding given the risk for viral transmission through breastmilk. ${ }^{66}$ If the desire to feed the infant breastmilk is identified prenatally, the options to obtain banked breastmilk from HIV-negative donors, identify a lactational surrogate, or to breastfeed herself in the context of maternal viral suppression and a neonatal surveillance plan could be discussed. Finally, arrangements should be made in the antepartum setting for acquiring neonatal zidovudine prophylaxis, though we acknowledge that there may be difficulties in acquiring this medication prior to birth. We recommend administration of zidovudine to all neonates exposed to HIV antenatally, with potential additional postexposure prophylaxis depending on risk factors for perinatal transmission (e.g., new acquisition of HIV during pregnancy).

\section{Conclusion}

Home birth in the midst of the COVID-19 pandemic is increasingly a topic of concern among pregnant persons in the United States, and especially among those who have comorbidities that may place them at high risk for complications associated with COVID-19 and birth in general. We have illustrated a person-centered, shared decision-making approach to counseling about and perinatal management of potential home birth for pregnant PLHIV, including risk mitigation strategies to allow for effective communication with hospital-based practitioners in case of clinical ques- tions or perinatal emergency. Most importantly, we recommend thoughtful communication to pregnant PLHIV about the extensive measures being taken in the hospital to protect all patients from COVID-19 and the thorough and compassionate care that they will receive in a hospital despite the pandemic. As COVID-19 continues to have an impact on the health care system in the United States, it is crucial that we, as health care providers, not only recognize the concerns that pregnant people have regarding their labor and delivery as well-informed and legitimate, but that we proactively create harm reduction strategies for health care delivery in high-risk obstetrical situations, such as home birth, that respond to the challenges posed by an ongoing pandemic.

Conflict of Interest

None declared.

\section{References}

1 Li Q, Guan X, Wu P, et al. Early transmission dynamics in Wuhan, China, of novel coronavirus-infected pneumonia. N Engl J Med 2020;382(13):1199-1207

2 Peeri NC, Shrestha N, Rahman MS, et al. The SARS, MERS and novel coronavirus (COVID-19) epidemics, the newest and biggest global health threats: what lessons have we learned? Int J Epidemiol 2020:dyaa033

3 Coronavirus disease 2019 (COVID-19): situation report-104. World Health Organization; 2020. Available at: https://www. who.int/docs/default-source/coronaviruse/situation-reports/ 20200503-covid-19-sitrep-104.pdf?sfvrsn=53328f46_2. Accessed May 3, 2020

4 Chen H, Guo J, Wang C, et al. Clinical characteristics and intrauterine vertical transmission potential of COVID-19 infection in nine pregnant women: a retrospective review of medical records. Lancet 2020;395(10226):809-815

5 Liu D, Li L, Wu X, et al. Pregnancy and perinatal outcomes of women with coronavirus disease (COVID-19) pneumonia: a preliminary analysis. AJR Am J Roentgenol 2020:1-6. Doi: 10.2214/AJR.20.23072

6 Liu Y, Chen H, Tang K, Guo Y. Clinical manifestations and outcome of SARS-CoV-2 infection during pregnancy. J Infect 2020;S01634453(20)30109-2

7 Zhu H, Wang L, Fang C, et al. Clinical analysis of 10 neonates born to mothers with 2019-nCoV pneumonia. Transl Pediatr 2020;9 (01):51-60

8 Li Y, Zhao R, Zheng S, et al. Lack of vertical transmission of severe acute respiratory syndrome coronavirus 2, China. Emerg Infect Dis 2020;26(06):26

9 Breslin N, Baptiste C, Miller R, et al. COVID-19 in pregnancy: early lessons. Am J Obstet Gynecol MFM 2020; (e-pub ahead of print). Doi: 10.1016/j.ajogmf.2020.100111

10 Zeng L, Xia S, Yuan W, et al. Neonatal early-onset infection with SARS-CoV-2 in 33 neonates born to mothers with COVID-19 in Wuhan, China. JAMA Pediatr 2020. Doi: 10.1001/jamapediatrics.2020.0878

11 Yu N, Li W, Kang Q et al. Clinical features and obstetric and neonatal outcomes of pregnant patients with COVID-19 in Wuhan, China: a retrospective, single-centre, descriptive study. Lancet Infect Dis 2020. Doi: 10.1016/S1473-3099(20)30176-6

12 Juusela A, Nazir M, Gimovsky M. Two cases of COVID-19 related cardiomyopathy in pregnancy. Am J Obstet Gynecol MFM 2020 (ePub ahead of print) . Doi: 10.1016/j.ajogmf.2020.100113

13 Iqbal SN, Overcash R, Mokhtari N, et al. An uncomplicated delivery in a patient with COVID-19 in the United States. N Engl J Med 2020;382(16):e34 
14 Breslin N, Baptiste C, Gyamfi-Bannerman C, et al. COVID-19 infection among asymptomatic and symptomatic pregnant women: two weeks of confirmed presentations to an affiliated pair of New York City hospitals. Am J Obstet Gynecol MFM 2020:100118. Doi: $10.1016 /$ j.ajogmf.2020.100118

15 Tekbali A, Grünebaum A, Saraya A, McCullough L, Bornstein E, Chervenak FA. Pregnant versus non-pregnant SARS-CoV-2 and COVID-19 hospital admissions: the first 4 weeks in New York. Am J Obstet Gynecol 2020; S0002-9378(20)30437-3 . Doi: 10.1016/j. ajog.2020.04.012

16 Chen L, Li Q, Zheng D, et al. Clinical characteristics of pregnant women with COVID-19 in Wuhan, China. N Eng J Med 2020. Doi: 10.1056/NEJMc2009226

17 Yan J, Guo J, Fan C, et al. Coronavirus disease 2019 (COVID-19) in pregnant women: A report based on 116 cases. Am J Obstet Gynecol 2020;S0002-9378(20)30462-2

18 Schnettler WT, Al Ahwel Y, Suhag A. Severe ARDS in COVID-19infected pregnancy: obstetric and intensive care considerations. Am J Obstet Gynecol MFM 2020. Doi: 10.1016/j.ajogmf.2020.100120

19 Hantoushzadeh S, Shamshirsaz AA, Aleyasin A, et al. Maternal death due to COVID-19 disease. Am J Obstet Gynecol 2020;S00029378(20)30516-0

20 Dong L, Tian J, He S, et al. Possible vertical transmission of SARSCoV-2 from an infected mother to her newborn. JAMA 2020

21 Zeng H, Xu C, Fan J, et al. Antibodies in infants born to mothers with COVID-19 pneumonia. JAMA 2020

22 Yee LM, Miller ES, Statton A, et al. Sustainability of statewide rapid HIV testing in labor and delivery. AIDS Behav 2018;22(02): 538-544

23 Dashraath P, Wong JLJ, Lim MXK, et al. Coronavirus disease 2019 (COVID-19) pandemic and pregnancy. Am J Obstet Gynecol 2020; S0002-9378(20)30343-4 . Doi: 10.1016/j.ajog.2020.03.021

24 Mullins E, Evans D, Viner RM, O’Brien P, Morris E. Coronavirus in pregnancy and delivery: rapid review. Ultrasound Obstet Gynecol 2020;55(05):586-592

25 Rasmussen SA, Smulian JC, Lednicky JA, Wen TS, Jamieson DJ. Coronavirus disease 2019 (COVID-19) and pregnancy: what obstetricians need to know. Am J Obstet Gynecol 2020;222(05): 415-426

26 Rasmussen SA, Jamieson DJ. Coronavirus disease 2019 (COVID19) and pregnancy: responding to a rapidly evolving situation. Obstet Gynecol 2020;135(05):999-1002

27 Di Mascio D, Khalil A, Saccone G, et al. Outcome of Coronavirus spectrum infections (SARS, MERS, COVID 1 -19) during pregnancy: a systematic review and meta-analysis. Am J Obstet Gynecol MFM 2020:100107

28 Della Gatta AN, Rizzo R, Pilu G, Simonazzi G. COVID19 during pregnancy: a systematic review of reported cases. Am J Obstet Gynecol 2020;S0002-9378(20)30438-5

29 Zaigham M, Andersson O. Maternal and perinatal outcomes with COVID-19: A systematic review of 108 pregnancies. Acta Obstet Gynecol Scand 2020

30 Fetters A. COVID-19 Is Upending Parents' Birth Plans. 2020. Available at: https://www.theatlantic.com/family/archive/2020/ 03/giving-birth-during-coronavirus-pandemic/608320/. Accessed March 22, 2020

31 New YHHBBPDC. New York Magazine, 2020. Available at: https:// www.thecut.com/2020/03/nyc-hospital-have-begun-banningvisitors-during-childbirth.html. Accessed March 29, 2020

32 COVID-19 Is No Reason to Abandon Pregnant People. Scientific American, 2020. Available at: https://blogs.scientificamerican com/observations/covid-19-is-no-reason-to-abandon-pregnantpeople/. Accessed March 29, 2020

33 Wax JR, Pinette MG, Cartin A, Blackstone J. Maternal and newborn morbidity by birth facility among selected United States 2006 lowrisk births. Am J Obstet Gynecol 2010;202(02):152.e1-152.e5

34 Wax JR, Lucas FL, Lamont M, Pinette MG, Cartin A, Blackstone J. Maternal and newborn outcomes in planned home birth vs planned hospital births: a metaanalysis. Am J Obstet Gynecol 2010;203(03):243.e1-243.e8

35 Snowden JM, Tilden EL, Snyder J, Quigley B, Caughey AB, Cheng YW. Planned out-of-hospital birth and birth outcomes. N Engl J Med 2015;373(27):2642-2653

36 Grünebaum A, McCullough LB, Sapra KJ, et al. Apgar's score of 0 at 5 minutes and neonatal seizures or serious neurologic dysfunction in relation to birth setting. Am J Obstet Gynecol 2013;209 (04):323.e1-323.e6

37 Grünebaum A, McCullough LB, Sapra KJ, et al. Early and total neonatal mortality in relation to birth setting in the United States, 2006-2009. Am J Obstet Gynecol 2014;211(04):390.e1-390.e7

38 Grünebaum A, McCullough LB, Brent RL, Arabin B, Levene MI, Chervenak FA. Perinatal risks of planned home births in the United States. Am J Obstet Gynecol 2015;212(03):350.e1-350. e6

39 Grünebaum A, McCullough LB, Orosz B, Chervenak FA. Neonatal mortality in the United States is related to location of birth (hospital versus home) rather than the type of birth attendant. Am J Obstet Gynecol 2020;S0002-9378(20)30067-3

40 Grünebaum A, McCullough LB, Arabin B, Dudenhausen J, Orosz B Chervenak FA. Underlying causes of neonatal deaths in term singleton pregnancies: home births versus hospital births in the United States. J Perinat Med 2017;45(03):349-357

41 Scrimshaw S, Backes EP. Committee on Assessing Health Outcomes by Birth S, et al. Birth Settings in America: Outcomes, Quality, Access, and Choice. Washington (DC): National Academies Press (US); 2020

42 Cheng YW, Snowden JM, King TL, Caughey AB. Selected perinatal outcomes associated with planned home births in the United States. Am J Obstet Gynecol 2013;209(04):325.e1-325.e8

43 Committee on Obstetric Practice. Committee opinion no. 697: planned home birth. Obstet Gynecol 2017;129(04):e117-e122

44 Watterberg KL; Committee on Fetus and Newborn. Policy statement on planned home birth: upholding the best interests of children and families. Pediatrics 2013;132(05):924-926

45 Patient-centered care for pregnant patients during the COVID-19 pandemic. 2020. Available at: https://s3.amazonaws.com/cdn. smfm.org/media/2279/homebirth.pdf. Accessed May 7, 2020

46 Ecker J, Minkoff H. Home birth: what are physicians' ethical obligations when patient choices may carry increased risk? Obstet Gynecol 2011;117(05):1179-1182

47 Wilbur MB, Little S, Szymanski LM. Clinical decisions. is home birth safe? N Engl J Med 2015;373(27):2683-2685

48 HOME BIRTH SAFETY ACT. 5 ILCS 80/4.402019. Available at: http://www.ilga.gov/legislation/BillStatus.asp?

DocNum $=2449 \&$ GAID $=15 \&$ DocTypeID $=$ HB\&LegId $=118457 \&$ SessionID $=108 \& G A=101$. Accessed May 7, 2020

49 Boelig RC, Saccone G, Bellussi F, Berghella V. MFM Guidance for COVID-19. Am J Obstet Gynecol MFM 2020. Doi: 10.1016/j. ajogmf.2020.100106

50 Labor and Delivery COVID-19 Considerations. 2020. Available at: https://s3.amazonaws.com/cdn.smfm.org/media/2319/SMFMSOAP_COVID_LD_Considerations_-_revision_4-14-20_PDF_(003). pdf. Accessed May 7, 2020

51 European Mode of Delivery Collaboration. Elective caesareansection versus vaginal delivery in prevention of vertical HIV-1 transmission: a randomised clinical trial. Lancet 1999;353 (9158):1035-1039

52 Sperling RS, Shapiro DE, Coombs RW, et al. Maternal viral load, zidovudine treatment, and the risk of transmission of human immunodeficiency virus type 1 from mother to infant. Pediatric AIDS clinical trials group protocol 076 study group. N Engl J Med 1996;335(22):1621-1629

53 Mofenson LM, Lambert JS, Stiehm ER, et al. Risk factors for perinatal transmission of human immunodeficiency virus type 1 in women treated with zidovudine. Pediatric AIDS clinical trials group study 185 team. N Engl J Med 1999;341(06):385-393 
54 Connor EM, Sperling RS, Gelber R, et al. Reduction of maternalinfant transmission of human immunodeficiency virus type 1 with zidovudine treatment. Pediatric AIDS clinical trials group protocol 076 study group. N Engl J Med 1994;331(18):1173-1180

55 Garcia PM, Kalish LA, Pitt J, et al; Women and Infants Transmission Study Group. Maternal levels of plasma human immunodeficiency virus type 1 RNA and the risk of perinatal transmission. $\mathrm{N}$ Engl J Med 1999;341(06):394-402

56 Briand N, Warszawski J, Mandelbrot L, et al; ANRS-EPF CO1-CO11 Study Group. Is intrapartum intravenous zidovudine for prevention of mother-to-child HIV-1 transmission still useful in the combination antiretroviral therapy era? Clin Infect Dis 2013;57 (06):903-914

57 Committee on Obstetric Practice; HIV Expert Work Group. ACOG committee opinion no. 751: labor and delivery management of women with human immunodeficiency virus infection. Obstet Gynecol 2018;132(03):e131-e137

58 Management of infants born to women with HIV infection: initial postnatal management of the neonate exposed to HIV. 2019. Available at: https://aidsinfo.nih.gov/guidelines/html/3/perinatal/188/initial-postnatal-management-of-the-neonate-exposedto-hiv. Accessed May 7, 2020

59 Craven C. Claiming respectable American motherhood: homebirth mothers, medical officials, and the state. Med Anthropol Q 2005;19(02):194-215

60 Craven CA. "Consumer's Right" to choose a midwife: shifting meanings for reproductive rights under neoliberalism. Am Anthropol 2007;109:701-712
61 Guidelines for the Use of Antiretroviral Agents in Adults and Adolescents Living With HIV. Department of Health and Human Services (HHS); 2017. Available at: https://aidsinfo.nih.gov/contentfiles/lvguidelines/adultandadolescentgl.pdf. Accessed May 7, 2020

62 Boucoiran I, Albert AYK, Tulloch K, et al. Human immunodeficiency virus viral load rebound near delivery in previously suppressed, combination antiretroviral therapy-treated pregnant women. Obstet Gynecol 2017;130(03):497-501

63 Cheyney M, Everson C, Burcher P. Homebirth transfers in the United States: narratives of risk, fear, and mutual accommodation. Qual Health Res 2014;24(04):443-456

64 Perinatal H. IV/AIDS. 2020. Available at: https://nccc.ucsf.edu/clinician-consultation/perinatal-hiv-aids/. Accessed March 22, 2020

65 Considerations for Antiretroviral Use in Special Patient Populations. Women with HIV. 2019. Available at: https://aidsinfo.nih. gov/guidelines/html/1/adult-and-adolescent-arv/23/womenwith-hiv. Accessed March 22, 2020

66 Levison J, Weber S, Cohan D. Breastfeeding and HIV-infected women in the United States: harm reduction counseling strategies. Clin Infect Dis 2014;59(02):304-309

67 Coronavirus (COVID-19) and pregnancy: what maternal-fetal medicine subspecialists need to know. 2020. Available at: https://s3.amazonaws.com/cdn.smfm.org/media/2262/COVID19_PDF.pdf. Accessed March 22, 2020

68 Caughey AB, Cheyney M. Home and birth center birth in the United States: time for greater collaboration across models of care. Obstet Gynecol 2019;133(05):1033-1050 